\title{
Procesos psicológicos básicos incluidos en publicaciones científicas sobre comportamiento alimentario en niños: revisión sistemática
}

\author{
Basic psychological processes included \\ in scientific publications on children \\ eating behavior: systematic review
}

Mónica del Pilar Díaz B. (1) Juan José Giraldo H. (2)

María Alejandra Forero C. (1)

\begin{abstract}
This research aimed to recognize which of the basic psychological processes are presented in the scientific literature of the last 11 years dedicated to analysis of eating behavior of children aged 5 to 12 years. A systematic review in Science Direct, Scopus, Scielo and Redalyc was conducted, with a final sample of 41 publications. Substantial research was identified in memory and perception, while few studies were found in decision-making and attention. The techniques most used were exposure to food and interview, while direct observation was detected less frequently. Age, gender and nutritional status of the participants were analyzed in $68.3 \%$ of the articles. Research in decision making and direct observation of everyday life is suggested as a priority. Key words: Feeding behavior, feeding, child, food consumption, child psychology (DeCS).
\end{abstract}

(1) Grupo de investigación en Alimentos, Nutrición y Salud. Departamento de Nutrición y Bioquímica, Facultad de Ciencias, Pontificia Universidad Javeriana, Bogotá, Colombia. (2) Universidad de la Sabana, Facultad de Psicología, Colombia.

Dirigir la correspondencia a: Profesora Mónica Díaz Beltrán Nutricionista Dietista, Magister en Administración Departamento de Nutrición y Bioquímica Facultad de Ciencias Pontificia Universidad Javeriana Nutrición y Salud, Bogotá-Colombia. E-mail: m-diazb@javeriana.edu.co mdiazbeltran@gmail.com

Este trabajo fue recibido el 7 de Marzo de 2014 y aceptado para ser publicado el 15 de Julio de 2014.

\section{INTRODUCCIÓN}

Actualmente, los hábitos alimentarios inadecuados constituyen un problema a nivel mundial; lo que es evidente en el creciente aumento de los problemas en salud relacionados con malnutrición. La estrategia mundial sobre régimen alimentario, actividad física y salud, para 2010 calculó 42 millones de niños en sobrepeso en todo el mundo, de los cuales 35 millones viven en países en desarrollo (1).

En este contexto la comprensión del comportamiento alimentario tiene un papel protagónico. El comportamiento alimentario se define como aquel conjunto de acciones vinculadas con la ingestión de alimentos, cuya motivación tiene una base biológica, psicológica y sociocultural (2). Entender esa base motivacional podrá generar estrategias mucho más efectivas que las planteadas hasta ahora.

Comprender el consumo de alimentos requiere el análisis de los cuatro procesos psicológicos básicos (percepción, atención, memoria y toma de decisiones), ya que estos participan en la formación del comportamiento, en este caso, frente a la alimentación en un individuo. Estos procesos son consecutivos, debido a que la percepción es la puerta de entrada de la información, la cual llega al cerebro y en el momento de convertirse en una actividad orientada hacia la focalización y filtro se da lugar a la atención, para posteriormente reconocer y fijar algunas características del alimento en la memoria, la cual permite recopilar dicha información en el presente para influir posteriormente en el proceso de toma de decisiones.

En este sentido, para reducir el espectro de indagación y estudio sobre la base motivacional que se relaciona con el comportamiento alimentario, se decidió explorar los procesos psicológicos básicos, las técnicas y las variables asociadas que han acompañado la investigación científica de la última década.

Procesos psicológicos básicos se asumen como aquellos aspectos más sencillos para explicar el comportamiento (3). Para el presente estudio, se definirán de acuerdo con literatura vigente para la percepción, la atención, la memoria y la toma de decisiones (tabla 1).

Con relación a lo que aquí se asumió, se entendió técnica como el procedimiento operativo para la recolección de datos. Las variables se tomaron como aquellas características que pueden ser percibidas o medidas, y que cambian de un sujeto a otro o en el transcurrir del tiempo (6).

Esta investigación bibliográfica tuvo por objetivo identifi- 
car en la literatura científica de los últimos 11 años los procesos psicológicos básicos, las técnicas y las variables asociadas con el análisis del comportamiento alimentario de escolares entre 5 y 12 años; para que pudiese reconocerse que tipo de análisis se han realizado hasta ahora en este campo de estudio.

\section{MÉTODOS}

Se realizó una búsqueda bibliográfica en las bases de datos Science Direct, Scopus, Scielo y Redalyc, incluyendo publicaciones desde enero de 2002 a marzo de 2013, en las cuales describían, caracterizaban o analizaban el comportamiento alimentario en escolares de 5 a 12 años de edad. La obtención de datos se cumplió a partir del uso de ocho descriptores de búsqueda en inglés, siete en español, los cuales fueron combinados con tres términos (Child, escolar y/o niño).

1. eating behavior

2. feeding practice

3. feeding behavior

4. dietary behavior

5. food behavior

6. food choice

7. meaning of food

8. meaning the size of the food

9. conducta alimentaria

10. hábitos alimentarios

11. prácticas alimentarias

12. consumo de alimentos

13. elección de la comida

14. significado de la comida

15. significado del tamaño de la comida

Para la búsqueda se definieron como criterios de exclusión:

1. Artículos de revisión narrativa o sistemática.

2. Artículos en los cuales el sujeto de investigación no era directamente el niño.

3. Artículos cuyos sujetos de investigación no tenían edades entre los 5 a 12 años.

Los títulos de los artículos fueron utilizados como primer filtro, excluyendo aquellos que tenían palabras como: lactancia materna, preescolares, adultos y alimentación embrionaria, nombres de enfermedades específicas, entre otras (figura 1). En base a lo anterior, se seleccionaron 416 artículos, de los cuales se leyó el resumen y se descartaron aquellos en donde se identificaron los criterios de exclusión anteriormente establecidos. Se obtuvieron 83 documentos, de los cuales no se seleccionaron 43 por las razones expuestas.

Con 41 artículos seleccionados, la información de los mismos fue compilada a través de fichas bibliográficas. A partir del análisis de contenido de éstas, se diseñó una matriz de variables, en la cual se registraba título del estudio, método, procesos psicológicos básicos incluidos y conclusiones a partir de dichos procesos.

Clasificada la información el análisis se basó en estadística descriptiva, se calcularon frecuencias relativas, se analizaron y se llegaron a conclusiones en cuanto a las tendencias de investigación en el tema.

\section{RESULTADOS}

Durante la búsqueda bibliográfica en las diferentes bases de datos seleccionadas, se identificó un alto número de publicaciones dirigidas a caracterizar el consumo por parte de escolares, en términos de diagnóstico de adecuación calórica y/o nutricional o grupos de alimentos consumidos. Los artículos incluidos debían realizar análisis en cuanto al comportamiento y tener en cuenta otras variables, adicionales al consumo, durante la investigación. En el Anexo 1 se listan los artículos finales seleccionados para este estudio.

Vale la pena mencionar que sólo $7 \%$ de las publicaciones incluidas $(n=41)$ corresponde a estudios latinoamericanos, los

TABLA 1

Descripción de procesos psicológicos básicos.

PROCESOS PSICOLOGICOS BÁSICOS

Percepción

Atención

Memoria

Toma de decisiones

\section{DEFINICIÓN / CRITERIOS DE ANÁLISIS}

Sensaciones individuales de sabor, apariencia, textura, olor y sonido, las cuales son producidas a partir de los estímulos captados por los sentidos (4).

Una vez generadas las sensaciones, este proceso involucra la selectividad, a partir de la cual se clasifican los estímulos sensoriales en aquellos que son relevantes y requieren ser examinados y monitoreados, y aquellos que son ignorados (5). Aquí se incluyen aspectos de fijación y/o preferencia.

Por medio de la percepción y la atención se crea una experiencia, pero es a través de la memoria que se logra fijar y reproducirla en una situación específica del presente. Por tanto la memoria se refiere a "la capacidad de codificar, almacenar y recuperar información" (5). Contiene comparaciones, recuerdos y asociaciones con situaciones o eventos previos.

"Proceso de seleccionar una opción preferida o el curso de una acción de entre un conjunto de alternativas" (5).

Fuente: Elaboración propia basada en las fuentes bibliográficas 4 y 5 . 
cuales son producto de investigaciones realizadas en Costa Rica, México y Chile respectivamente. De ahí, que existe un campo de acción importante en el tema, en aras de aportar al desarrollo de estrategias regionales que generen modificaciones sostenibles en los hábitos alimentarios y por tanto repercutan de manera positiva en el ámbito de la salud pública.

Aunque en ninguna de las investigaciones, los autores mencionaron de manera directa los procesos psicológicos básicos, éstos se identificaron mediante el análisis de los resultados, a partir de los criterios establecidos en el apartado metodológico. Como consecuencia se identificó que 18 de los artículos seleccionados evaluaban la percepción, tres de ellos la atención, 33 la memoria y 10 la toma de decisiones, un mismo artículo podía contener más de un proceso psicológico básico. En el caso del proceso de memoria, es el que aparece con mayor frecuencia en los artículos seleccionados (tabla 2).

Un ejemplo de la inclusión de más de una proceso psicológico en los artículos bajo análisis, se halla en la investigación de Ureña en 2009 (7), en la cual se aplicó un cuestionario a escolares costarricenses, evaluó sensaciones individuales de sabor, olor y apariencia de verduras y frutas principalmente (percepción), reconocimiento de determinados alimentos (memoria) y la elección de qué comer en los comedores escolares (toma de decisiones).

\section{FIGURA 1}

Criterios de inclusión y exclusión de los artículos hallados.

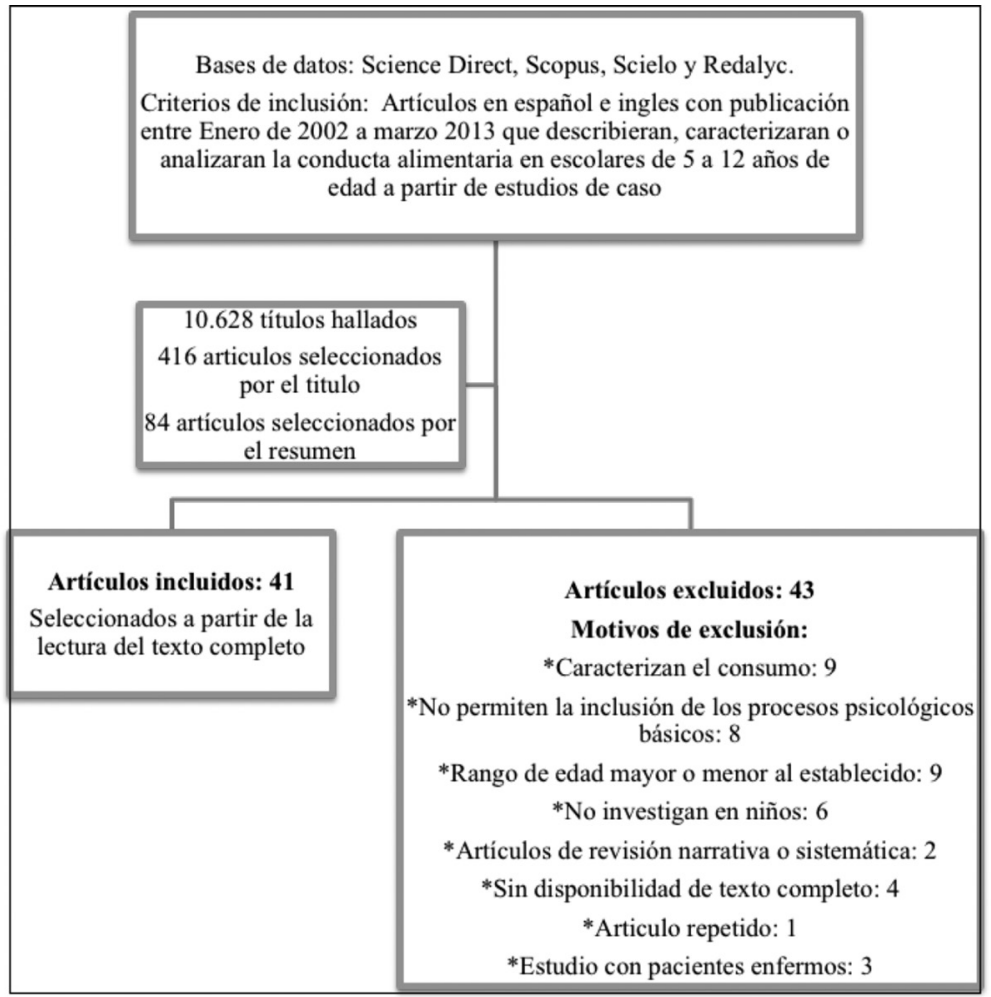

Fuente: Elaboración propia.

TABLA 2

Número de artículos que incluyen análisis del comportamiento alimentario con percepción, atención, memoria y toma de decisiones.

\begin{tabular}{lcccc} 
& Percepción & Atención & Memoria & Toma de decisiones \\
Número de artículos & 18 & 3 & 33 & 10 \\
\hline Fuente: Elaboración propia. & & & & \\
\hline
\end{tabular}


El proceso de memoria (figura 2) fue incluido en el análisis de la aceptación de un alimento tras la familiaridad desarrollada cuando eran expuestos a educación nutricional (8), al alimento $(9,10)$ a logos o imágenes publicitarias $(11)$ o a características de un alimento (12). En este proceso se destaca la profundización en experiencias previas, en características organolépticas, conocimientos de cualidades nutricionales y recuerdos con base en la publicidad de alimentos.

En menor proporción fueron evaluados la toma de de- cisiones, mediante el consumo de alimentos en comedores escolares (13) o al presentarle diferentes opciones de alimentos (14); y atención, que fue estudiado mediante la clasificación de alimentos en un listado $(15,16)$.

Respecto a las condiciones bajo las cuales se obtuvieron los resultados, la mitad de los artículos revisados utiliza metodología experimental (figura 3), bajo condiciones como educación, exposición directa al alimento y juegos para la obtención de la información. La metodología menos utili-

FIGURA 2

Procesos psicológicos básicos evaluados en los 41 artículos seleccionados.

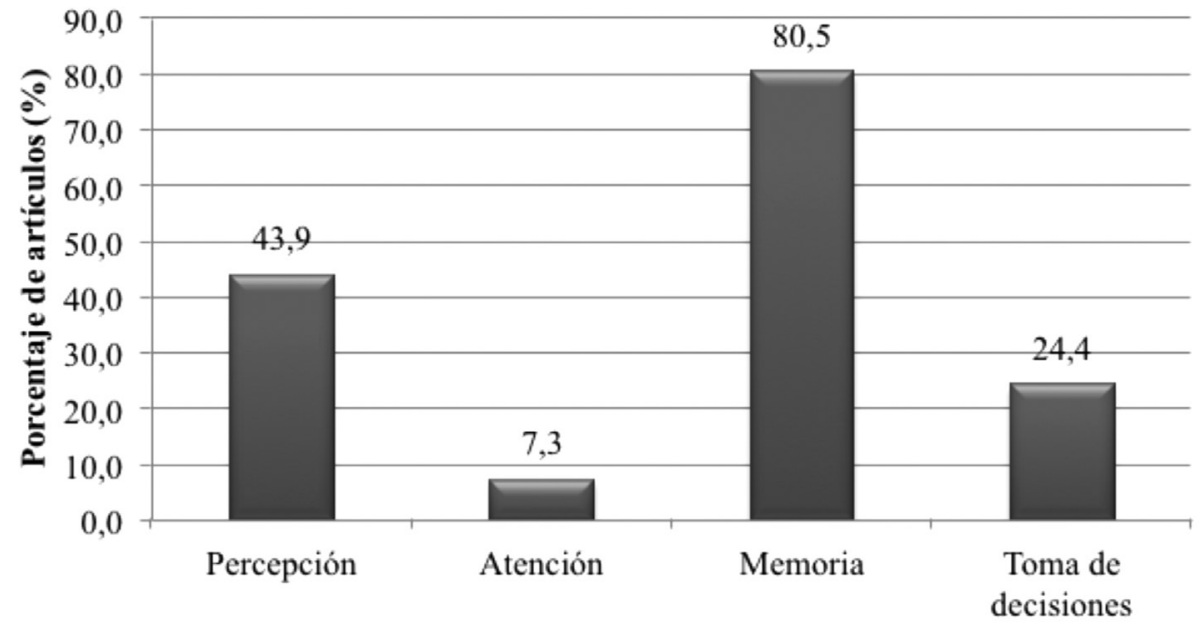

Fuente: Elaboración propia.

FIGURA 3

Porcentaje de condiciones bajo las cuales se obtuvo la información.

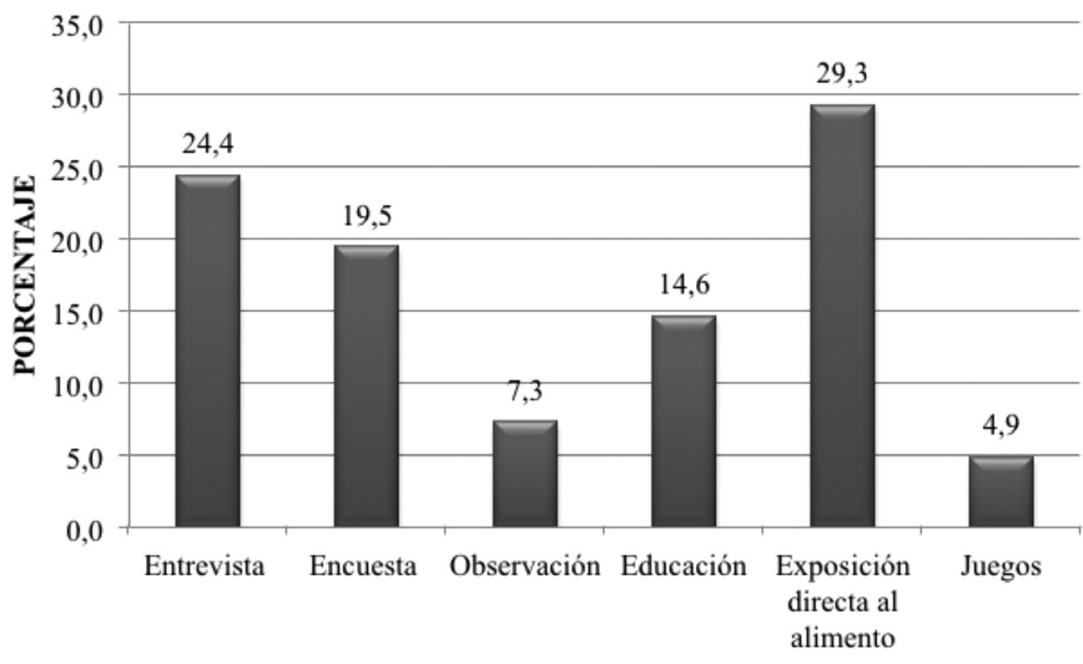


zada correspondió con la observación directa, en la cual se analizaron reacciones oro-faciales (17) o si el comportamiento alimentario de los niños variaba cuando estaban solos o con compañía (18).

Cuando se cruzaron los procesos y las condiciones (figura 4), se observó que la memoria, el proceso más frecuente, fue incluido en investigaciones que utilizaron en mayor proporción las encuestas (27,3\%) y las entrevistas (21,2\%), que los juegos $(3,0 \%)$ y las observaciones $(6,1 \%)$ en los niños participantes.

Adicional a lo anterior, se hallaron tres variables de investigación comúnmente relacionadas con el análisis del comportamiento alimentario: género, edad y estado nutricional. Cuando se sumaron los diversos análisis que recurren a estas tres variables, el $68,3 \%$ de los artículos incluidos en la presente revisión tuvieron en cuenta al menos una de dichas variables (figura 5). Género fue la variable más utilizada de manera aislada o junto a la edad y el estado nutricional (46,3\%) y solo uno de los artículos seleccionados incluyó el análisis de las tres variables (19).

\section{DISCUSIÓN}

Con los resultados encontrados, se puede indicar una escasa profundización en los análisis que incluyen atención y toma de decisiones en el comportamiento alimentario de escolares. Si bien hay suficiente información sobre las características percibidas por los escolares en cuanto a determinados alimentos, como es el caso de la identificación de verduras como amargas (20) y alimentos dulces como agradables (21) o los recuerdos que evocan de tiempos de comida (22) donde relacionan ciertos alimentos con tiempos de fiestas (memoria), aún hay mucho que investigar sobre la manera como el proceso de atención se relaciona con la ingesta de los alimentos y finalmente cómo eligen un curso de acción frente a los mismos.

El escaso análisis del proceso de atención en los artículos seleccionados puede estar relacionado con la evidencia dis- ponible de los hábitos alimentarios de la población infantil, la cual hoy día muestran un alto consumo de alimentos de bajo valor nutricional (23). Como consecuencia de ello, los estudios pueden no estar focalizándose en aquellos alimentos que tienen un agrado especial por el individuo y aquellos que ignoran, pues ya están identificados. Sin embargo, valdría la pena profundizar en cuáles son las características que Ilaman más la atención para tomar la decisión de consumir o no aquellos alimentos que prefiere o rechaza.

El análisis de la toma de decisiones es un aspecto de gran relevancia en términos de la salud de la población, debido a que es precisamente el alto y/o bajo consumo de determinados alimentos, lo cual se vuelve un factor de riesgo frente alteraciones de crecimiento y desarrollo en los niños, así como para la aparición de enfermedades crónicas no transmisibles en la población adulta. De ahí, que investigar en la decisión es lo que permitirá saber qué pasa con el consumo después de determinadas intervenciones o bajo qué parámetros se decide un curso de acción frente a la alimentación.

Resulta interesante develar como autores que realizaron educación alimentaria y nutricional en sus investigaciones resaltan que, a pesar de lograr cambios inmediatos en el consumo de alimentos, estos no son sostenidos a través del tiempo (9). De acuerdo con esos resultados, investigar en la decisión versus la variable tiempo daría cuenta de la eficacia real de las intervenciones, en términos de cambios de hábitos alimentarios inadecuados y adherencia a un estilo de vida saludable.

En relación con las condiciones de trabajo, es paradójico observar que la más frecuente fue la exposición al alimento, mientras que la observación se utilizó menos. Esto debido a que muchos de los trabajos analizaban la reacción del niño frente un alimento, pero no consideraban el consumo de alimentos en la cotidianidad desde la observación, sino bajo condiciones controladas tanto en laboratorios, como en

FIGURA 4

Condiciones bajo las cuales se obtuvo la información para cada proceso psicológico básico.

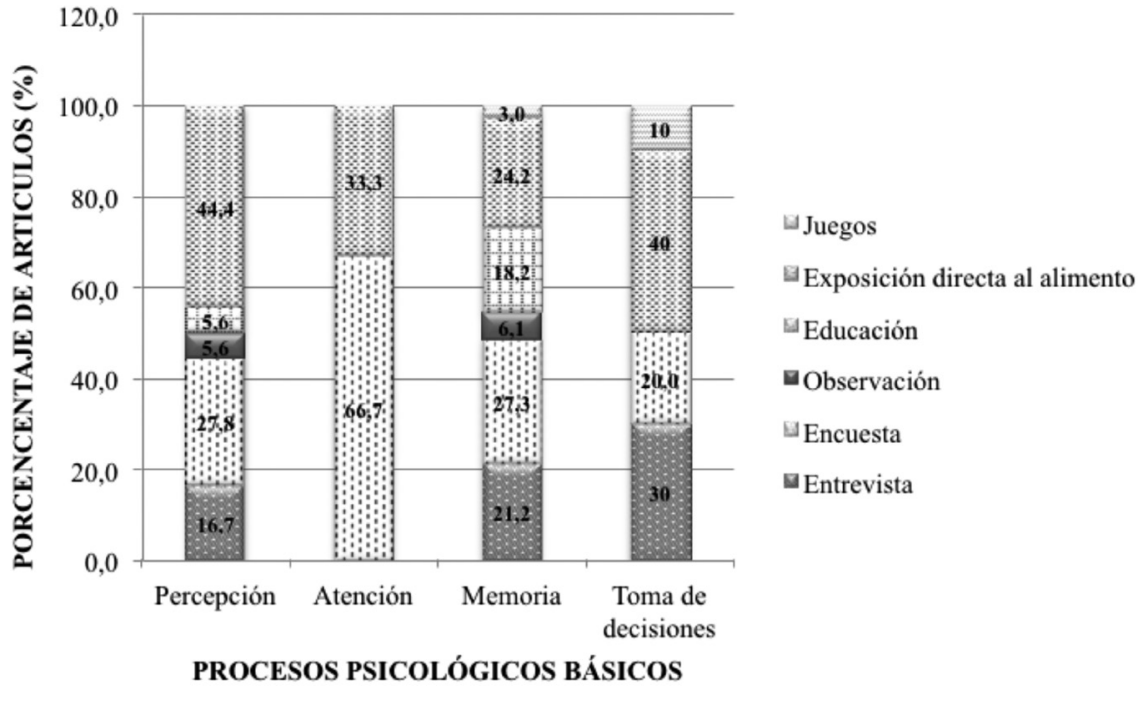


otros ambientes donde se habían dispuesto alimentos. Como consecuencia de ello, mucha de la información obtenida no necesariamente da un registro de los hábitos de consumo y más cuando vemos que la segunda condición utilizada es la entrevista, pues es un hecho que no necesariamente el individuo reporta lo que pasa realmente con relación a la ingestión de alimentos. Indudablemente en el contexto del proceso trabajado en mayor medida: la memoria, la entrevista es una excelente herramienta para obtener información de eventos previos.

Al discriminar condiciones por proceso psicológico evaluado, se observó como en aquellos en los que se ha profundizado más se han utilizado diferentes técnicas. No obstante, las empleadas son acordes a lo que se está buscando, ejemplo de esto es indagar percepción con una entrevista o combinar esta con la sensación posterior a consumir el alimento. Sin embargo, en el caso de la toma de decisiones, adicional al número limitado de investigaciones que tienen en cuenta ese proceso, llama la atención que $50 \%$ de los estudios en ese proceso lo hagan a través de la entrevista más que de la visualización directa, pues nuevamente es el comportamiento reportado, que no necesariamente es igual al real.

Finalmente, en cuanto a variables asociadas con la investigación en el tema, las cuales a saber fueron: género, estado nutricional y edad, la combinación de estas tres sólo se observó en un artículo, lo cual es un aspecto a tener en cuenta en futuras investigaciones dado que las intervenciones alimentarias se hacen en niños con edades particulares y características nutricionales específicas. En consecuencia de ello explorar el comportamiento en función de las diferencias por cada una de las tres variables permitiría intervenciones más precisas.

\section{CONCLUSIONES}

La literatura científica de los últimos once años viene estudiando el comportamiento alimentario de los escolares, desde una perspectiva comportamental, que permite la inclusión de los procesos psicológicos básicos (percepción, atención, memoria y toma de decisiones) en su análisis, a pesar de no ser variables literalmente expuestas en el documento.

El análisis de los 41 artículos seleccionados, identificó que los procesos psicológicos básicos en mayor medida evaluados por los investigadores fueron memoria y percepción. Como consecuencia de ello es posible concluir que hay un gran camino que recorrer en términos de lo que llama la atención de los niños y cómo toman decisiones frente a los alimentos. Lo anterior requiere de un abordaje urgente, dadas las condiciones de salud de la población y las cifras alarmantes de problemáticas que conciernen al consumo de alimentos. Sobre todo en América Latina donde los estudios en cualquiera de los cuatro procesos son escasos.

En la actualidad, diferentes estrategias como "cinco al día", "estrategia mundial sobre régimen alimentario, actividad física y salud", Guías Alimentarias Nacionales, entre otras, buscan generar un cambio en los hábitos alimentarios de la población, sin embargo, se mantiene el incremento de los índices de malnutrición con sus respectivos problemas en salud pública.

Es en este punto, el trabajo a nivel interdisciplinar exalta su importancia, debido a que no es suficiente saber cuál es el consumo de alimentos de las personas para trabajar en pro de un cambio. Conocer como los procesos psicológicos básicos condicionan el comportamiento alimentario, permitirá influir desde la percepción que se tiene de un alimento hasta el momento de decidir el consumo de la opción más saludable.

Es prioridad investigar en tomas de decisiones y en la observación directa de la cotidianidad, si se quiere saber cómo se elige un curso de acción frente a los alimentos, teniendo en cuenta que son precisamente esas decisiones cotidianas las que ponen en riesgo la salud la población.

\section{FIGURA 5}

Porcentaje de artículos que incluyeron en su análisis edad, género y estado nutricional.

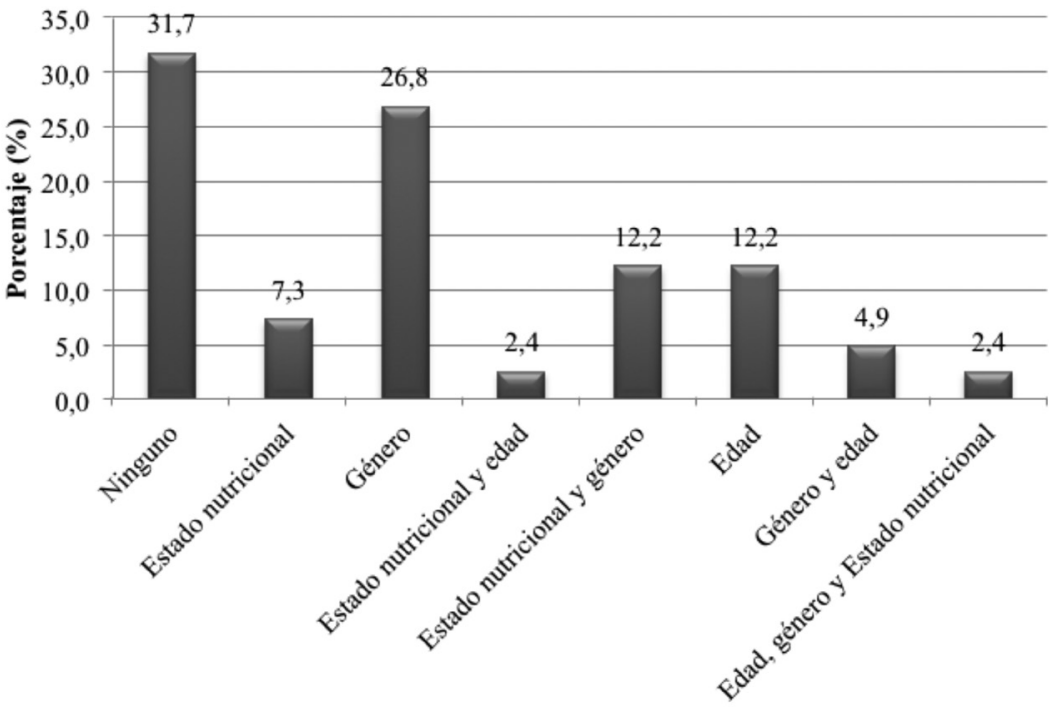




\section{RESUMEN}

Esta investigación tuvo por objetivo reconocer cuáles de los procesos psicológicos básicos están presentes en publicaciones científicas de los últimos 11 años dedicadas al análisis del comportamiento alimentario de niños entre 5 y 12 años. Se realizó una revisión sistemática en Science Direct, Scopus, Scielo y Redalyc, con una muestra final de 41 artículos. Se identificó una mayor investigación en memoria y percepción, frente a una indagación escasa en la toma de decisiones y atención. Las técnicas utilizadas en mayor medida fueron la exposición al alimento y la entrevista, mientras que la observación directa se presentó en menor frecuencia. La edad, el género y el estado nutricional de los participantes fueron variables analizadas en $68,3 \%$ de los artículos. Se sugiere como prioridad la investigación en tomas de decisiones y la observación directa de la cotidianidad.

Palabras clave: Conducta alimentaria, alimentación, niño, consumo de alimentos, psicología Infantil (DeCS)

Conflicto de intereses: Los autores declaran no tener ningún tipo de conflicto de intereses.

\section{BIBLIOGRAFÍA}

1. Organización Mundial de la Salud. Sobrepeso y Obesidad Infantil. Available at: http://www.who.int/dietphysicalactivity/childhood/es/. Accessed Febrero 4, 2013.

2. Santacoloma A, Quiroga L. Perspectivas de estudio de la conducta alimentaria. Rev Iberoam Psicol Ciencia y Tecnol. 2009;2(2):7-15.

3. Pérez V, Gutiérrez MT, García A, Gómez J. Procesos psicológicos básicos. Un análisis funcional. Madrid: Person Educación 2005: p 80-81.

4. Rubinstein J. L. editor. Principios de psicología general. México D.F.: Grijalbo; 1967: p 272.

5. Wilson R, Keil Feditors. The Mit Encyclopedia of the Cognitive Sciences. Cambridge, Massachusetts; 1999;514:220.

6. Infante $C$ editor. Guía para la presentación de proyectos de investigación. Bogotá - Colombia: Universidad Nacional de Colombia 2010.

7. Ureña M. Razones de consumo de frutas y vegetales en escolares costarricenses de una zona urbana. Rev Costar Salud Púb. 2009;18(1):15-21.

8. Fahlman MM, Dake JA, McCaughtry N, Martin J. A pilot study to examine the effects of a nutrition intervention on nutrition knowledge, behaviors, and efficacy expectations in middle school children: Research article. J Sch Health 2008;78(4):216-22.

9. Schindler JM, Corbett D, Forestell CA. Assessing the effect of food exposure on children's identification and acceptance of fruit and vegetables. Eating Behav 2013;14(1):53-56.

10. Heim S, Stang J, Ireland M. A Garden Pilot Project Enhances Fruit and Vegetable Consumption among Children. J Am Diet Assoc. 2009 7;109(7):1220-6.

11. Arredondo $E$, Castaneda D, Elder JP, Slymen D, Dozier D. Brand name logo recognition of fast food and healthy food among children. J Community Health 2009;34(1):73-78.

12. Atik D, Ozdamar Ertekin Z. Children's perception of food and healthy eating: Dynamics behind their food preferences. Int. J. Cons Stud. 2013;37(1):59-65.

13. Théodore $F$, Bonvecchio A, Blanco I, Irizarry L, Nava A, Carriedo A. Significados culturalmente construidos para el consumo de bebidas azucaradas entre escolares de la Ciudad de México. Rev Pan. Sal Púb. 2011;30(4):327-34.

14. Liem DG, Zandstra LH. Children's liking and wanting of snack products: Influence of shape and flavour. Int J Beh. Nutr Phys Act. 2009;6.

15. Kopelman CA, Roberts LM, Adab P. Advertising of food to children: Is brand logo recognition related to their food knowledge, eating behaviours and food preferences? J. Pub Health 2007;29(4):358-67.

16. Rollins BY, Loken E, Birch LL. Stability and change in snack food likes and dislikes from 5 to 11 years. Appetite 2010;55(2):371-3.

17. Soussignan $R$, Schaal B, Boulanger $V$, Gaillet $M$, Jiang $T$. Orofacial reactivity to the sight and smell of food stimuli. Evidence for anticipatory liking related to food reward cues in overweight children. Appetite 2012;58(2):508-16.

18. Salvy S, Coelho JS, Kieffer E, Epstein LH. Effects of social contexts on overweight and normal-weight children's food intake. Physiol Behav 2007 12/5;92(5):840-6.

19. Keller KL, Kuilema LG, Lee N, Yoon J, Mascaro B, Combes $A-$, et al. The impact of food branding on children's eating behavior and obesity. Phys Beh. 2012;106(3):379-86.

20. Havermans RC, Jansen A. Increasing children's liking of vegetables through flavour-flavour learning. Appetite 2007;48(2):259-62.

21. Kuntz MG, Fiates GM, Teixeira E. Healthy and tasty school snacks: Suggestions from Brazilian children consumers. Int. J. Cons. Stud. 2012;36(1):38-43.

22. Warren $E$, Parry $O$, Lynch $R$, Murphy S. 'If I don't like it then I can choose what I want': Welsh school children's accounts of preference for and control over food choice. Health Promot Internat. 2008;23(2):144-51.

23. Holsten JE, Deatrick JA, Kumanyika S, Pinto-Martin J, Compher CW. Children's food choice process in the home environment. A qualitative descriptive study. Appetite 2012 2;58(1):64-73.

\section{ANEXO 1}

Listado de artículos incluidos.

\section{REFERENCIA BIBLIOGRÁFICA}

Théodore F, Bonvecchio A, Blanco I, Irizarry L, Nava A, Carriedo A. Significados culturalmente construidos para el consumo de bebidas azucaradas entre escolares de la ciudad de méxico. Revista Panamericana de Salud Pública. 2011;30(4):327-334

Protudjer JLP, Marchessault G, Kozyrskyj AL, Becker AB. Children's perceptions of healthful eating and physical activity. Canadian Journal of Dietetic Practice and Research. 2010;71(1):19-23

Arredondo E, Castaneda D, Elder JP, Slymen D, Dozier D. Brand name logo recognition of fast food and healthy food among children. J Community Health. 2009;34(1):73-8

Salvy S, Coelho JS, Kieffer E, Epstein LH. Effects of social contexts on overweight and normal-weight children's food intake. Physiol Behav. 2007 12/5;92(5):840-6 
Orrell-Valente JK, Hill LG, Brechwald WA, Dodge KA, Pettit GS, Bates JE. "Just three more bites": An observational analysis of parents' socialization of children's eating at mealtime. Appetite. 2007 1;48(1):37-45.

Soussignan R, Schaal B, Boulanger V, Gaillet M, Jiang T. Orofacial reactivity to the sight and smell of food stimuli. evidence for anticipatory liking related to food reward cues in overweight children. Appetite. 2012;58(2):508-16.

Fahlman MM, Dake JA, McCaughtry N, Martin J. A pilot study to examine the effects of a nutrition intervention on nutrition knowledge, behaviors, and efficacy expectations in middle school children: Research article. J Sch Health. 2008;78(4):216-22

Schindler JM, Corbett D, Forestell CA. Assessing the effect of food exposure on children's identification and acceptance of fruit and vegetables. Eating Behav. 2013;14(1):53-6

Matvienko O. Impact of a nutrition education curriculum on snack choices of children ages six and seven years. Journal of Nutrition Education and Behavior. 2007 $0 ; 39(5): 281-5$.

Heim S, Stang J, Ireland M. A garden pilot project enhances fruit and vegetable consumption among children. J Am Diet Assoc. 2009 7;109(7):1220-6.

Gillis L. Use of an interactive game to increase food acceptance - A pilot study. Child: Care, Health and Development. 2003;29(5):373-5.

Ratcliffe MM, Merrigan KA, Rogers BL, Goldberg JP. The effects of school garden experiences on middle school-aged students' knowledge, attitudes, and behaviors associated with vegetable consumption. Health promotion practice. 2011;12(1):36-43

Osborne CL, Forestell CA. Increasing children's consumption of fruit and vegetables: Does the type of exposure matter? Physiology and Behavior. 2012;106(3):362-8.

Zeinstra GG, Koelen MA, Kok FJ, de GraafC. Children's hard-wired aversion to pure vegetable tastes. A 'failed' flavour-nutrient learning study. Appetite. 2009;52(2):528-30.

Laureati M, Pagliarini E, Mojet J, Köster EP. Incidental learning and memory for food varied in sweet taste in children. Food Quality and Preference. 2011;22(3):264-70.

Morizet D, Depezay L, Combris P, Picard D, Giboreau A. Effect of labeling on new vegetable dish acceptance in preadolescent children. Appetite. 2012;59(2):399-402.

Havermans RC, Jansen A. Increasing children's liking of vegetables through flavour-flavour learning. Appetite. 2007;48(2):259-62.

Greenhalgh J, Dowey AJ, Horne PJ, Fergus Lowe C, Griffiths JH, Whitaker CJ. Positive- and negative peer modelling effects on young children's consumption of novel blue foods. Appetite. 2009;52(3):646-53.

Rollins BY, Loken E, Birch LL. Stability and change in snack food likes and dislikes from 5 to 11 years. Appetite. 2010;55(2):371-3.

Keller KL, Kuilema LG, Lee N, Yoon J, Mascaro B, Combes A-, Deutsch B, Sorte K, Halford JCG. The impact of food branding on children's eating behavior and obesity. Physiology and Behavior. 2012;106(3):379-86.

Grubliauskiene A, Verhoeven M, Dewitte S. The joint effect of tangible and non-tangible rewards on healthy food choices in children. Appetite. 2012;59(2):403-8.

Liem DG, Zandstra LH. Children's liking and wanting of snack products: Influence of shape and flavour. International Journal of Behavioral Nutrition and Physical Activity. $2009 ; 6$.

Salvy S, Vartanian LR, Coelho JS, Jarrin D, Pliner PP. The role of familiarity on modeling of eating and food consumption in children. Appetite. 2008 0;50(2-3):514-8.

Jansen E, Mulkens S, Jansen A. Do not eat the red food!: Prohibition of snacks leads to their relatively higher consumption in children. Appetite. 2007;49(3):572-7.

Olsen A, Ritz C, Kramer L, Møller P. Serving styles of raw snack vegetables. what do children want? Appetite. 2012;59(2):556-62.

Kopelman CA, Roberts LM, Adab P. Advertising of food to children: Is brand logo recognition related to their food knowledge, eating behaviours and food preferences? Journal of Public Health. 2007;29(4):358-67.

Castells Cuixart. M, Capdevila Prim. C, Girbau Solá. T, Rodriguez Caba. C. Estudio del comportamiento alimentario en escolares de 11 a 13 años de barcelona. Revista Nutrición Hospitalaria. 2006;21(4):511-516.

McKinley MC, Lowis C, Robson PJ, Wallace JMW, Morrissey M, Moran A, Livingstone MBE. It's good to talk: Children's views on food and nutrition. Eur J Clin Nutr. 2005;59(4):542-51.

Farrow CV. Do parental feeding practices moderate the relationships between impulsivity and eating in children? Eating Behav. 2012 4;13(2):150-3.

Tuorila H, Mustonen S. Reluctant trying of an unfamiliar food induces negative affection for the food. Appetite. 2010;54(2):418-21.

Michels N, Sioen I, Braet C, Eiben G, Hebestreit A, Huybrechts I, Vanaelst B, Vyncke $K$, De Henauw S. Stress, emotional eating behaviour and dietary patterns in children. Appetite. 2012;59(3):762-9.

Warren E, Parry O, Lynch R, Murphy S. 'If I don't like it then I can choose what I want': Welsh school children's accounts of preference for and control over food choice. Health Promot Internation. 2008;23(2):144-51.

Ureña M. Razones de consumo de frutas y vegetales en escolares costarricenses de una zona urbana. Revista Costarrisense de Salud Pública. 2009;18(1):15-21.

Théodore F, Bonvecchio A, Blanco I, Carreto Y. Representaciones sociales relacionadas con la alimentación escolar: El caso de las escuelas públicas de la ciudad de méxico. Salud Colectiva. 2011;7(2):215-229.

Edwards JSA, Hartwell HH. Fruit and vegetables - attitudes and knowledge of primary school children. Journal of Human Nutrition and Dietetics. 2002;15(5):365-74.

Olivares S, Bustos N, Moreno X, Lera L, Gortez S. Actitudes y prácticas sobre alimentación y actividad física en niños obesos y sus madres en santiago, chile. Revista Chilena de Nutrición. 2006;33(2):170-179.

Zeinstra GG, Koelen MA, Kok FJ, de Graaf C. Cognitive development and children's perceptions of fruit and vegetables; a qualitative study. International Journal of Behavioral Nutrition and Physical Activity. 2007;4.

Dammann K, Smith C. Food-related attitudes and behaviors at home, school, and restaurants: Perspectives from racially diverse, urban, low-income 9- to 13-year-old children in minnesota. Journal of Nutrition Education and Behavior. 2010;42(6):389-97.

Atik D, Ozdamar Ertekin Z. Children's perception of food and healthy eating: Dynamics behind their food preferences. International Journal of Consumer Studies. 2013;37(1):59-65.

Kuntz MG, Fiates GM, Teixeira E. Healthy and tasty school snacks: Suggestions from brazilian children consumers. International Journal of Consumer Studies. 2012; 36(1):38-43

Holsten JE, Deatrick JA, Kumanyika S, Pinto-Martin J, Compher CW. Children's food choice process in the home environment. A qualitative descriptive study. Appetite. $2012 ; 58(1): 64-73$ 